\title{
Hypofractionated radiotherapy after conservative surgery for breast cancer: analysis of acute and late toxicity
}

\author{
Letizia Deantonio ${ }^{1}$, Giuseppina Gambaro ${ }^{1}$, Debora Beldì , Laura Masini', Sara Tunesi ${ }^{1}$, Corrado Magnani², \\ Marco Krengli $i^{*}$
}

\begin{abstract}
Background: A variety of hypofractionated radiotherapy schedules has been proposed after breast conserving surgery in the attempt to shorten the overall treatment time. The aim of the present study is to assess acute and late toxicity of using daily fractionation of 2.25 Gy to a total dose of 45 Gy to the whole breast in a monoinstitutional series.

Methods: Eighty-five women with early breast cancer were assigned to receive 45 Gy followed by a boost to the tumour bed. Early and late toxicity were scored according to the Radiation Therapy Oncology Group criteria. For comparison, a group of 70 patients with similar characteristics and treated with conventional fractionation of 2 Gy to a total dose of $50 \mathrm{~Gy}$ in 25 fractions followed by a boost, was retrospectively selected.

Results: Overall median treatment duration was 29 days for hypofractionated radiotherapy and 37 days for conventional radiotherapy. Early reactions were observed in 72/85 (85\%) patients treated with hypofractionation and in $67 / 70(96 \%)$ patients treated with conventional fractionation $(p=0.01)$. Late toxicity was observed in 8 patients (10\%) in the hypofractionation group and in 10 patients (15\%) in the conventional fractionation group, respectively $(p=0.4)$.
\end{abstract}

Conclusions: The hypofractionated schedule delivering 45 Gy in 20 fractions shortened the overall treatment time by 1 week with a reduction of skin acute toxicity and no increase of late effects compared to the conventional fractionation. Our results support the implementation of hypofractionated schedules in clinical practice.

\section{Background}

Radiotherapy (RT) reduces the risk of local relapse and breast cancer mortality [1] and is offered to nearly all patients after conservative surgery and to selected patients after mastectomy. The international standard RT regimen after breast conservative surgery for early breast cancer delivers 25 daily fractions of 2 Gy to a total dose of 50 Gy over 5 weeks followed by 5 fractions of 2 Gy as a boost to the tumour bed [2]. The high number of women with breast cancer, receiving postoperative RT, led to think that a shorter course of irradiation would result in improved quality of life for

\footnotetext{
* Correspondence: krengli@med.unipmn.it

'Department of Radiotherapy, University Hospital Maggiore della Carità, Novara, Italy

Full list of author information is available at the end of the article
}

patients, in potentially better integration with systemic treatments and in reduced costs. Therefore, alternative schedules based on a lower total dose delivered in fewer, larger fractions (hypofractionation) were firstly introduced in Canada and the United Kingdom (UK) $[3,4]$. The Canadian randomised trial [3] tested 42.5 Gy in 16 fractions against 50 Gy in 25 fractions. Results suggested equivalence in terms of local control and breast cosmetic results for the 16-fractions regimen.

The two most recent randomized studies [5,6] were conducted by the START Trials in order to test the effects of radiotherapy schedules using fraction size larger than 2.0 Gy. The START Trial A tested two dose levels of a 13-fractions regimen delivered over 5 weeks and the START Trial B compared 40 Gy in 15 fractions of 2.67 Gy in 3 weeks with a control group of 50 Gy in
C Biomed Central

(c) 2010 Deantonio et al; licensee BioMed Central Ltd. This is an Open Access article distributed under the terms of the Creative Commons Attribution License (http://creativecommons.org/licenses/by/2.0), which permits unrestricted use, distribution, and reproduction in any medium, provided the original work is properly cited. 
25 fractions of 2.0 Gy over 5 weeks. These studies seem to offer rates of late adverse effects and local-regional tumour relapse at least as favourable as the standard schedule.

The aim of the present study carried out in a monoinstitutional clinical setting is to assess acute and late toxicity of hypofractionated radiotherapy after conservative surgery using a regimen of $2.25 \mathrm{~Gy} /$ fraction to a total dose of $45 \mathrm{~Gy}$ to the whole breast followed by a boost comparing the results with those of a similar group of patients treated with conventional fractionation schedule.

\section{Methods}

\section{Patients}

From January 2006 to January 2008, 85 patients with invasive carcinoma of the breast treated with conservative surgery and biopsy of sentinel lymph node or axillary lymph node dissection were prospectively treated with whole breast irradiation of 45 Gy in 20 fractions, $2.25 \mathrm{~Gy} /$ fraction, followed by 9 Gy in 3 fractions to the tumour bed as a boost dose. Eligibility criteria were: age $\geq 60$ years, $\mathrm{T} \leq 2 \mathrm{~cm}$, negative surgical margins and no indication to lymph node RT ( $\leq 3$ positive lymphnodes). Patients with history of contralateral breast cancer, multifocal disease, serious non-malignant disease (e.g. cardiovascular or pulmonary), severe mental or physical disorders were excluded from the study. The initial work-up included chest radiogram, liver ultrasound, bone scan, full blood count, kidney and liver function tests. Written informed consent was obtained from patients before start treatment following the rules of our institution.

A second group of 70 patients with similar characteristics in terms of clinical history, staging and type of surgery was retrospectively selected from patients treated since 2005 with post-operative breast RT with conventional schedule of 50 Gy in 25 fraction, 2 Gy/fraction followed by $10 \mathrm{~Gy}$ in 5 fractions to the tumour bed as a boost dose.

\section{Radiotherapy}

All patients underwent post-operative RT planned using the three-dimensional treatment planning system, Pinnacle (Philips, Eindhoven, The Netherlands). Computed Tomography (CT) images were obtained by helical CT (Prospeed, General Electric Medical Systems, Milwaukee, WI), covering the entire thoracic region from the apex of the lung to the diaphragm, with patients in treatment position. Target and non-target volumes were outlined according to the criteria of the International Commission of Radiation Units (ICRU) and Measurements Reports 50 and $62[7,8]$. The clinical target volume (CTV) was defined as the entire palpable breast tissue starting $5 \mathrm{~mm}$ below the skin. The planning target volume (PTV) was obtained by adding $10 \mathrm{~mm}$ margin to the CTV, except in the direction of the skin. Ipsilateral lung was automatically outlined from the apex to the base and the left ventricle was manually outlined in case of left sided cancer. The treatment technique consisted of two opposed tangential fields by using 6-18 MV photon beams. Radiation fields were appropriately customized by multileaf collimator when needed in order to spare the surrounding healthy tissues. The angle of the beams was adjusted to minimize the irradiation of lung parenchyma and left ventricle. Appropriate physical wedge compensation was used to ensure a uniform dose distribution throughout the target volume. The total dose prescribed to the ICRU point was $45 \mathrm{~Gy}$, delivered with 2.25 Gy per fraction, 5 days a week. A boost dose of 9 Gy in 3 fractions was given using a 6-9 MeV electron field, depending on the depth of the original tumor site. Dose calculation with a grid of $3 \mathrm{~mm}$ was performed using the collapsed cone convolution algorithm of the treatment planning system, including the correction for tissue heterogeneity. For each patient, dosevolume histograms (DVHs) for target, lung and left ventricle for left-sided cancers were calculated. The same technique had been used for the patients treated with conventional radiotherapy to a total dose of $50 \mathrm{~Gy}$, 2 Gy/fraction and a boost dose of 10 Gy in 5 fractions to the tumour bed.

Biologically effective dose (BED) was calculated assuming alpha/beta ratio equals to 10 Gy for early reactions, 3 Gy for late reactions. In the group assigned to receive $45 \mathrm{~Gy}$ to the whole breast, BED was $55 \mathrm{~Gy}$ for early effects, 78 Gy for late effects versus 60 Gy for early effects, 83 Gy for late effects in the group treated with 50 Gy.

Sequential chemotherapy (cyclophosphamide - methotrexate -5-fluorouracil, doxorubicin - cyclophosphamide, 5-fluorouracil - epirubicin - cyclophosphamide) and hormone therapy given concomitantly to radiotherapy (tamoxifen or aromatase inhibitors) were given in 20/85 patients (24\%) in the hypofractionation group while $65 /$ 85 patients $(76 \%)$ received hormone therapy alone. The patients of the group treated by conventional fractionation received sequential chemotherapy in 20/70 cases (28\%) and hormone therapy concomitant to radiotherapy in 50/70 cases (71\%).

Early and late toxicity were scored according to the Radiation Therapy Oncology Group/European Organization for Research and Treatment Cancer (RTOG/ EORTC) criteria in both groups of patients [9].

\section{Statistical analysis}

The chi-square test and Fisher's exact test were used to compare the two treatment groups. 
The association of early and late toxicity with breast volume, maximum radiation dose and chemotherapy was analyzed in the two treatment groups using Chisquare test and logistic regression. The Chi-square test was used for comparing acute and late toxicity between the patients treated with hypofractionation and those treated with conventional RT and for comparing the frequency of breast volume $<500 \mathrm{cc}$ and $\geq 500 \mathrm{cc}$ in the two treatment groups. Logistic regression was used to adjust the effect on toxicity of covariates, such as breast volume, maximum dose and chemotherapy. Chi-square test for trend was used to compare acute toxicity between the patients treated with hypofractionation and those treated with conventional RT.

The t-test was used to compare breast volume between the patients treated with hypofractionation and those treated with conventional RT.

Survival curves were obtained to show the cumulative probability of experiencing adverse effects during 6 months follow-up interval. The actuarial occurrence of late toxicity was calculated by the Kaplan-Meier method and the two treatment groups were compared using the Log-rank test.

Cox's proportional hazards regression model was fitted in order to obtain the hazard ratio (HR) for hypofractionation adjusted by volume and chemotherapy. A $p$ value of less than 0.05 was considered to be statistically significant. Statistical analysis was performed using the SAS package version 8.02 (SAS Institute, Inc, Cary, NC, USA).

\section{Results}

Both treatment groups were comparable in terms of age, performance status, tumour stage, adjuvant chemotherapy and hormone treatment. Differences were observed for lymph node stage, breast volume and breast maximum dose (Table 1).

Median time from surgery to RT was 29 days for hypofractionated RT and 37 days for conventional RT. No patient interrupted the treatment.

The mean follow-up was 15.0 months (median12.6; $25^{\text {th }}$ quartile 7.8 and $75^{\text {th }}$ quartile 20.8 months) in the hypofractionation RT group and 28.6 months (median32.2; $25^{\text {th }}$ quartile 22.1 and $75^{\text {th }}$ quartile 40.0 months) in the conventional fractionation group.

\section{Acute toxicity}

Early reactions, consisting in skin erythema, were observed in 72 patients $(85 \%)$ in the hypofractionation group and in 67 (95\%) in the conventional RT group (chi-square $\mathrm{p}=0.01$; chi-square test for trend $\mathrm{p}=0.001$ ) (Table 2).

A significant correlation by chi-square test $(p=0.001)$ between breast volume and maximum dose was found for the occurrence of acute toxicity (Figure 1). Adjuvant treatments did not influence acute toxicity.

Logistic regression analysis was carried on for adjusting for potential confounders. In this analysis, acute skin toxicity was classified in two categories: mild (G0 and G1) and severe (G2 and G3). Hypofractionation reduced the risk of severe acute toxicity: odd ratio (OR) adjusted for volume was 0.45 (95\% CI $=0.23-0.93)$. Including in the analyses also maximum breast dose and chemotherapy did not provide a significant contribution to the model fit.

\section{Late toxicity}

Late toxicity was assessed after 6 months in 76/85 patients in the hypofractionation group and in $67 / 70$ patients in the standard RT group since 9 and 3 patients, respectively, were lost at follow-up and were not included in the statistical analysis. Late toxicity according to the RTOG criteria was observed in 8 patients $(10 \%)$ in the hypofractionation group and in 10 patients $(15 \%)$ in the conventional fractionation group (Table 3). The difference was not statistically significant (chi-square $\mathrm{p}=0.4$ ). Cumulative occurrence of late toxicity over time was analyzed using Kaplan-Meier method and compared by log-rank test, resulting not statistically significant $(\mathrm{p}=0.17$ ) (Figure 2). At 12 and 30 months, the risk of late toxicity was $5.9 \%$ and $29.2 \%$ in the group treated by hypofractionation, $8.2 \%$ and $10.6 \%$ in the group treated by standard RT, respectively.

Cox's proportional hazards regression analysis showed that hypofractionation, adjusted by volume and chemotherapy, was not associated with the hazard of late toxicity $(\mathrm{HR}=2.16 ; 95 \% \mathrm{CI}=0.68-6.84 ; \mathrm{p}=0.19)$. In the same model, we observed that breast volume increased the hazard of late toxicity over time $(\mathrm{HR}=$ 1.27; $95 \% \mathrm{CI}=1.04-1.55 ; \mathrm{p}=0.016$ ).

\section{Discussion}

Although a number of preliminary data support the use of partial breast irradiation in low risk patients [10], whole breast irradiation will probably remain the standard treatment for intermediate and high risk cases. Breast irradiation after conservative surgery is usually given daily for 5-6 weeks. Results of many trials showed that shorter fractionation schedules are as effective as the conventional schedule of $50 \mathrm{~Gy}$ in 25 fractions in terms of preventing recurrence of cancer in the breast [3-5].

The linear-quadratic model is typically used to calculate the biologically equivalent dose taking into account a larger dose per fraction over a shorter period of time [11]. As a matter of fact, the size of dose per fraction may influence the tolerance of normal tissues and also the therapeutic results [12]. 
Table 1 Patients' baseline characteristics.

\begin{tabular}{|c|c|c|c|c|c|}
\hline & \multicolumn{2}{|c|}{ Hypofractionation No. } & \multicolumn{2}{|c|}{ Conventional fractionation No. } & \multirow{2}{*}{$\frac{\mathbf{P}}{0.688}$} \\
\hline Age & $\geq 60$ & & $\geq 60$ & & \\
\hline Mean & 71.9 & & 71.6 & & \\
\hline IQR & 9 & & 11 & & \\
\hline KPS & & & & & 0.742 \\
\hline 100 & 34 & $40 \%$ & 26 & $37 \%$ & \\
\hline 90 & 51 & $60 \%$ & 44 & $63 \%$ & \\
\hline T stage & & & & & 0.054 \\
\hline 1 & 70 & $82 \%$ & 49 & $70 \%$ & \\
\hline 2 & 14 & $17 \%$ & 21 & $30 \%$ & \\
\hline 3 & 1 & $1 \%$ & 0 & $0 \%$ & \\
\hline $\mathrm{N}$ stage & & & & & 0.045 \\
\hline 0 & 69 & $83 \%$ & 47 & $67 \%$ & \\
\hline 1 & 16 & $17 \%$ & 23 & $33 \%$ & \\
\hline Histologic type & & & & & 0.357 \\
\hline Ductal & 66 & $77 \%$ & 54 & $77 \%$ & \\
\hline Lobular & 13 & $15 \%$ & 7 & $10 \%$ & \\
\hline Others & 6 & $8 \%$ & 9 & $13 \%$ & \\
\hline Histologic grade & & & & & 0.781 \\
\hline 1 & 17 & $20 \%$ & 13 & $18 \%$ & \\
\hline 2 & 48 & $56 \%$ & 37 & $54 \%$ & \\
\hline 3 & 20 & $24 \%$ & 20 & $28 \%$ & \\
\hline Chemotherapy & & & & & 0.375 \\
\hline yes & 19 & $22 \%$ & 20 & $29 \%$ & \\
\hline no & 66 & $88 \%$ & 50 & $71 \%$ & \\
\hline Hormone therapy & & & & & 0.457 \\
\hline yes & 66 & $78 \%$ & 50 & $71 \%$ & \\
\hline no & 19 & $22 \%$ & 20 & $29 \%$ & \\
\hline Breast volume & & & & & 0.039 \\
\hline$\geq 500 \mathrm{cc}$ & 24 & $28 \%$ & 29 & $41 \%$ & \\
\hline$<500 \mathrm{cc}$ & 61 & $72 \%$ & 41 & $59 \%$ & \\
\hline Breast maximum dose & & & & & 0.0009 \\
\hline$>107 \% * *$ & 26 & $36 \%$ & 40 & $65 \%$ & \\
\hline$\leq 107 \%$ & 59 & $64 \%$ & 30 & $35 \%$ & \\
\hline Surgery & & & & & 0.584 \\
\hline Quadrantectomy & 84 & $99 \%$ & 68 & $97 \%$ & \\
\hline Tumorectomy & 1 & $1 \%$ & 2 & $7 \%$ & \\
\hline
\end{tabular}

$\mathrm{IQR}=$ interquartile range

KPS $=$ Karnofsky performance status

$\mathrm{T}=$ tumor

$\mathrm{N}=$ lymph-node

** $>53.5$ Gy for conventional fractionation and > 48.1 Gy for hypofractionation

$\mathrm{P}=$ chi-square test or Fisher's exact test

This model predicts that the normal tissue toxicity is not increased when the fraction dose is modestly increased and the total dose is reduced accordingly to the linear-quadratic formalism [11].

In the present study women with more than 60 years were enrolled. In literature, some authors used age as a selection criteria [13] while other studies, like START trial, considered eligible women aged over 18 years.
As far as the incidence and the grade of acute skin toxicity are concerned, the present study showed a significant difference $(\mathrm{p}=0.01)$ between the two treatment groups with lower toxicity in the hypofractionation group. A similar finding was reported in a Japanese retrospective study [14] in which the authors observed that acute toxicity by hypofractionated RT (40 Gy in 16 fractions, fraction size $2.5 \mathrm{~Gy}$ ) was milder than that by the conventional schedule 
Table 2 Acute radiation reactions (RTOG scale)

\begin{tabular}{|c|c|c|c|c|c|}
\hline Grade & Hypofractionation & & $\begin{array}{l}\text { Conventional } \\
\text { fractionation } \\
\end{array}$ & & $p$ \\
\hline & No & $\%$ & No & $\%$ & \\
\hline 0 & 13 & 16 & 3 & 4 & $<0.001$ \\
\hline 1 & 51 & 60 & 34 & 49 & \\
\hline 2 & 19 & 22 & 29 & 41 & \\
\hline 3 & 2 & 2 & 4 & 6 & \\
\hline
\end{tabular}

(50 Gy in 25 fractions, fraction size 2 Gy) ( $p=0.01$ ). Other literature studies reported substantially similar results. Whelan et al. [3] in a randomized trial of 1234 women with early stage negative nodes breast cancer, comparing conventional fractionation (50 Gy in 25 fractions) versus hypofractionation (42.5 Gy in 16 fractions) found no difference regarding the early toxicity between the two regimens. Substantially similar results were reported by Olivotto et al. [15] in a non randomized study on 186 patients treated with 44 Gy in 16 fractions over 22 days. The authors reported results to be comparable to their historical patients. No significant differences in acute skin reaction were showed also in an Egyptian study [13] analysing 30 patients randomized to receive adjuvant $\mathrm{RT}$ with conventional schedule or hypofractionation of 42.5 Gy in 16 fractions.

The present study showed a significant difference in volume size ( $p=0.039)$ between the two patient groups and an association between severity of acute effects and breast volume $(p=0.001)$. In order to overcome

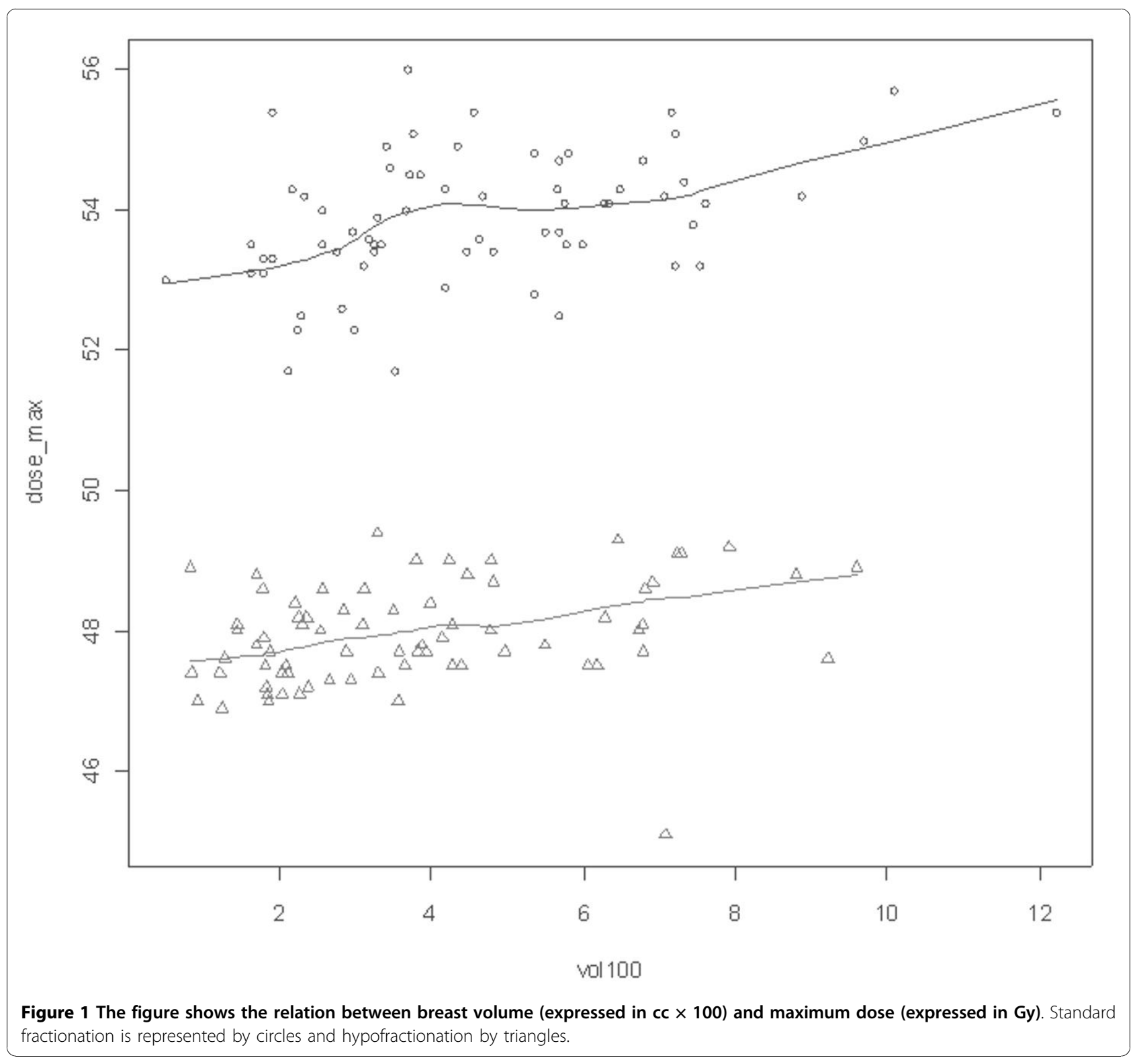


Table 3 Late radiation reactions (RTOG scale).

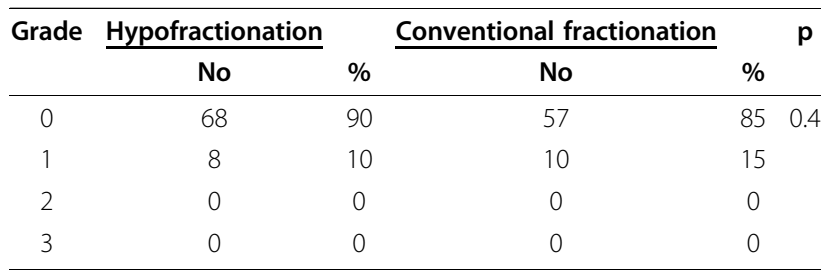

confounding analyses were adjusted by breast volume. In this regard, the Egyptian study showed also a significant correlation between breast volume and severity of acute skin reactions. In the present study, systemic adjuvant treatment had not significant correlation with toxicity as reported also in other literature series [12].

The reduction of acute toxicity in patients treated with hypofractionated RT in the present series might be explained by the BED value of acute reaction calculated by linear quadratic model that was lower than that of the conventional schedule and by the breast volume that was larger in the group of conventional fractionation $(p=0.039)$. As a matter of fact, large volume breasts are frequently associated with dose inhomogeneity and this fact may influence the occurrence of acute reactions. In fact, the present study found that voluminous breasts had more frequently a maximum dose higher than the cut off of 48.1 and 53.5 Gy, i.e. the $107 \%$ of prescribed dose for hypofractionation and standard RT, respectively (Table 1).

Similarly to what reported in other literature studies, no statistical difference $(\mathrm{p}=0.4)$ for late toxicity was found between patients treated by hypofractionation and those treated by conventional fractionation [9]. However, the UK randomised trial [4] on hypofractionated radiotherapy using fraction sizes of 2,3 and $3.3 \mathrm{~Gy}$ in 1410 randomized patients found that the 3.3 Gy schedule to a total dose of 42.9 Gy allowed to obtained the worst cosmetic result meaning that late effects may worsen when the fraction size is largely increased. In our series, the actuarial occurrence of late toxicity between the two groups, by Kaplan-Meier, resulted not significant $(\mathrm{p}=0.17)$. At 12 months and 30 months, the risk of late toxicity was $5.9 \%$ and $29.2 \%$ respectively in the group treated by hypofractionation, $8.7 \%$ and $10.6 \%$ in the group treated by standard RT (Figure 2). Similarly to what observed for acute toxicity's results, breast volume increased the risk of late toxicity $(\mathrm{p}=0.016)$ when analysed with Cox's proportional hazards regression model.

Main limitations of the present study were the nonrandomized design and consequently the possible presence of some minor bias such as the different breast size, the different percentage of patients with maximum dose higher than cut off in the two groups and the

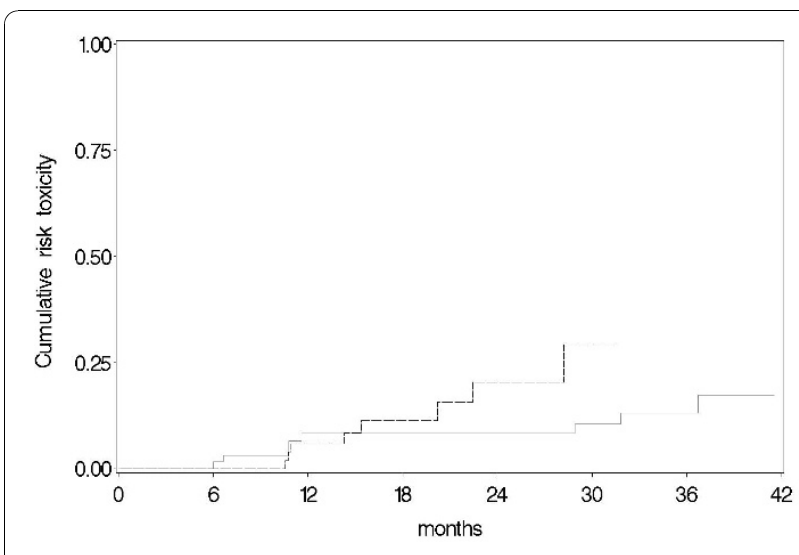

Figure 2 Time course of breast fibrosis as a cumulative risk of late toxicity in patients treated with standard RT (continuous line) and patients treated with hypofractionation (dotted line).

relatively shorter follow-up time in the hypofractionation group that could affect the incidence of late side effects. Nevertheless, statistical adjustment made possible to reach a conclusion in respect to the association of fractionation and adverse effects.

\section{Conclusions}

The data reported in the present study confirm the feasibility of the hypofractionated RT with 2.25 Gy per fraction to a total dose of $45 \mathrm{~Gy}$ in patients with invasive breast cancer in daily practice. Patients well tolerated the treatment with excellent compliance and nobody stopped the radiotherapy course that lasted 8 days less than that of conventional fractionation.

Acute dermatitis by hypofractionation was milder than that by the conventional RT $(\mathrm{p}=0.01)$. No significant difference of late effects $(\mathrm{p}=0.4)$ compared to the conventional schedule was found. These results, like those from other literature studies, support the implementation of hypofractionated radiation schedules in clinical practice.

\section{Acknowledgements}

This work was supported by a grant from the "Lega Italiana per la lotta contro i tumori", Section of Novara, Italy.

\section{Author details}

'Department of Radiotherapy, University Hospital Maggiore della Carità Novara, Italy. ${ }^{2}$ Department of Epidemiology and Biostatistics, University Hospital Maggiore della Carità, Novara, Italy.

\section{Authors' contributions}

LD was the study coordinator, participated in the development of the study and drafted the manuscript. CM and ST worked on analysis of data, GG, DB and LM participated in the design of the study and were involved in continuing optimization. MK was the study chairman, developed the design of the study, was involved in continuing optimization and helped to draft the manuscript. All authors read and approved the final manuscript. 


\section{Competing interests}

The authors declare that they have no competing interests.

Received: 3 September 2010 Accepted: 23 November 2010

Published: 23 November 2010

\section{References}

1. Clarke M, Collins R, Darby S, Early Breast Cancer Trialists' Collaborative Group (EBCTCG): Effects of radiotherapy and of differences in the extent of surgery for early breast cancer on local recurrence and 15-year survival: an overview of randomized trials. Lancet 2005, 366:2087-06.

2. Fisher B, Anderson $\mathrm{S}$, Redmond R, Wolmark DL, Cronin W: Re-analysis and results after 12 years of follow-up in a randomized clinical trial comparing total mastectomy with lumpectomy with or without irradiation in the treatment of breast cancer. N Engl J Med 1995, 333:1456-61.

3. Whelan TJ, MacKenzie R, Julian J, Levine M, Shelley W, Grimard L, Lada B, Lukka H, Perera F, Fyles A, Laukkanen E, Gulavita S, Benk V, Szechtman B: Randomized trial of breast irradiation schedules after lumpectomy for women with lymph node-negative breast cancer. J Natl Cancer Inst 2002, 94(15):1143-50.

4. Owen JR, Ashton A, Bliss JM, Homewood J, Harper C, Hanson J, Haviland J, Bentzen SM, Yarnold JR: Effect of radiotherapy fraction size on tumour control in patients with early-stage breast cancer after local tumour excision: long-term results of a randomised trial. Lancet Oncol 2006, 7:467-71.

5. The START Trialists' Group: The UK Standardisation of Breast Radiotherapy (START) Trial A of radiotherapy hypofractionation for treatment of early breast cancer: a randomised trial. Lancet 2008, 9:331-41.

6. The START Trialists' Group: The UK Standardisation of Breast Radiotherapy (START) Trial B of radiotherapy hypofractionation for treatment of early breast cancer: a randomised trial. Lancet 2008, 371:1098-17.

7. International Commission of Radiation Units and Measurements: ICRU Report 50: Prescribing, recording, and reporting photon beam therapy. Bethesda, MD: International Commission of Radiation Units and Measurements; 1993. International Commission of Radiation Units and Measurements.

8. ICRU Report 62: Prescribing, recording, and reporting photon beam therapy (supplement to ICRU Report 50). Bethesda, MD: International Commission of Radiation Units and Measurements; 1999.

9. Cox JD, Stetz J, Pajak TF: Toxicity criteria of the Radiation Therapy Oncology Group (RTOG) and the European Organization for Research and Treatment of Cancer (EORTC). Int I Radiat Oncol Biol Phys 1995, 31:1341-46.

10. Formenti SC: External-Beam partial breast irradiation. Semin Radiat Oncol 2005, 15:92.

11. Fowler JF: The linear-quadratic formula and progress in fractionated radiotherapy. Br J Radiol 1989, 62(740):679-94

12. Plataniotis GA, Dale RG: FIPEM., FRCR. Biologically effective dose-response relationship for breast cancer treated by conservative surgery and postoperative radiotherapy. Int I Radiat Oncol Biol Phys 2009, 75:512-7.

13. Taher AN, El-Baradie MM, Essa H, Zaki O, Ezzat S: Hypofractionation versus conventional fractionation radiotherapy after conservative treatment of breast cancer: Early Skin Reactions and Cosmetic Results. J. of the Egyptian Nat. Cancer Inst 2004, 16:178-187.

14. Osako T, Oguchi M, Kumada M, Nemoto K, Iwase T, Yamashita T: Acute radiation dermatitis and pneumonitis in Japanese breast cancer patients with whole breast hypofractionated radiotherapy compared to conventional radiotherapy. Jpn J Clin Oncol 2008, 38:334-338.

15. Olivotto IA, Weir LM, Kim-Sing C, Bajdik CD, Trevisan CH, Doll CM: Late cosmetic results of short fractionation for breast conservation. Radiother Oncol 1996, 41:7-13.

doi:10.1186/1748-717X-5-112

Cite this article as: Deantonio et al:: Hypofractionated radiotherapy after conservative surgery for breast cancer: analysis of acute and late toxicity. Radiation Oncology 2010 5:112.

\section{Submit your next manuscript to BioMed Central and take full advantage of:}

- Convenient online submission

- Thorough peer review

- No space constraints or color figure charges

- Immediate publication on acceptance

- Inclusion in PubMed, CAS, Scopus and Google Scholar

- Research which is freely available for redistribution

Submit your manuscript at www.biomedcentral com/submit 